\section{La comunicación para la salud como disciplina en las universidades estadounidenses}

Rina Alcalay ${ }^{1}$
En los Estados Unidos de América, el área de las comunicaciones asociada con la salud ha sido objeto de estudio por más de 20 años. En casi todas las instituciones académicas, se dedica a esta disciplina una división o área de interés. Hay, además, dos revistas que tratan exclusivamente del tema: Health Communication y Journal of Health Communication. Sin embargo, son pocas las universidades que han establecido un plan de estudios de especialización en ese campo. De hecho, solo una, la Escuela de Comunicaciones de la Universidad Emerson en conjunto con la Escuela de Medicina Tufts, en Boston, ofrece un título académico específicamente en comunicación y salud. En ese programa, la disciplina se define como "el arte y la técnica de informar, influenciar y motivar a los individuos, las instituciones y el público general sobre temas de salud importantes. Entre esos temas se encuentran la prevención de enfermedades, la promoción de la salud, las políticas de salud, el financiamiento y el mejoramiento de la calidad de vida y salud de los miembros de una comunidad" (1).

El origen de esta área de especialización en comunicaciones para la salud se atribuye a un proyecto en particular, el Stanford Heart Disease Prevention Project [Proyecto para Prevenir las Enfermedades del Corazón] dirigido por el doctor Jack Farquahar, cardiólogo, y el doctor Nathan Maccoby, profesor de comunicaciones $(2,3)$. Reconociendo la importancia de esta área de estudio, el gobierno estadounidense estableció en 1993 la Oficina de Comunicación y Salud ubicada en los Centros para el Control y la Prevención de Enfermedades (CDC). Los CDC definen la comunicación para la salud como "el diseño y la diseminación de mensajes y estrategias que se basan en la investigación del consumidor, para promover la salud de los individuos y las comunidades" (4).

\section{La importancia de la comunicación para promover la salud}

La importancia de la comunicación en el ámbito de la salud es clara. Existe una disparidad entre los avances logrados por la medicina y el conocimiento y la aplicación de estos por el público. Mientras que los profesionales de la salud tienen grandes conocimientos sobre la prevención de las enfermedades y la promoción de la salud, no saben necesa- 
riamente cómo comunicar efectivamente esa información tan vital para la sociedad. Esta situación constituye el foco central de interés del área de comunicación para la salud, es decir, el estudio de la naturaleza y la función de los medios necesarios para hacer que los temas de salud lleguen y produzcan un efecto en las audiencias objetivo (5). Dentro del ámbito del quehacer de la comunicación para la salud se consideran a) la calidad de la comunicación interpersonal en locales de salud, por ejemplo, entre el médico y el paciente; b) la comunicación entre miembros de una organización, por ejemplo, en instituciones de salud; y c) el alcance de los medios de comunicación masiva así como el diseño, la ejecución y la evaluación de campañas de comunicación.

El impacto de la promoción de salud en los Estados Unidos ha sido muy positivo y con frecuencia se ven en la prensa ejemplos de esa influencia educativa. Por ejemplo, los datos estadísticos de 1996 muestran que, como resultado de los programas de prevención y promoción, las muertes por sida han disminuido notablemente, la natalidad en adolescentes ha comenzado a descender y la esperanza de vida ha aumentado al nivel máximo de 76,1 años (6). Además, como resultado de intensas campañas en los medios de comunicación, entre 1996 y 1997 la mortalidad por cáncer de mama tuvo una reducción importante en el estado de California. Esa reducción se atribuye al alto porcentaje de mujeres que, como resultado de las campañas de educación, se hicieron mamografías. En consecuencia, los tumores de mama que antes se diagnosticaban cuando alcanzaban un tamaño promedio de $3 \mathrm{~cm}$, ahora se detectan al alcanzar los $2 \mathrm{~cm}(7)$.

Estos datos contrastan con otros divulgados en los medios de comunicación, como los que se refieren a Hungría, donde los hábitos de vida malsanos como el fumar e ingerir alcohol y una dieta dañina han afectado notablemente a la salud de la población. Según las estadísticas del Banco Mundial, la esperanza de vida de los hombres húngaros es la más baja de Europa después de Rusia. Solo la mitad de los hombres de Hungría llegarán a los 70 años de vida, ya que el porcentaje de cáncer entre ellos es el más alto del mundo, uno de cada cinco puede describirse como alcohólico y las tasas de cirrosis equivalen a 14 veces las de Suecia. Expertos internacionales en salud reprochan al gobierno de Hungría por no haber puesto en marcha campañas masivas de comunicación en salud (7).

Por otro lado Polonia, que a comienzos del decenio de los noventa se encontraba en el mismo estado de salud catastrófico que el de Hungría, ha mejorado espectacularmente sus tasas de esperanza de vida. Los investigadores médicos de ese país lo atribuyen exclusivamente a cambios en la dieta y disminución del hábito de fumar como consecuen- cia de enérgicas campañas de promoción de la salud. Las muertes por enfermedades del corazón han disminuido de 15 a $20 \%$ en la segunda mitad de los años noventa (7).

\section{La investigación sobre comunicación para la salud}

En los Estados Unidos, el campo de las investigaciones sobre la comunicación para la salud tiene tres características principales:

- Es un área de investigación aplicada. El área de la comunicación para la salud es muy halagadora para cualquier persona que se interese por las ciencias sociales aplicadas. Provee un contexto excelente para poner a prueba y aplicar teorías y principios sobre la comunicación social. Su aporte al desarrollo del conocimiento está precisamente en probar y aplicar en el terreno principios basados en la comunicación interpersonal, grupal, organizacional, de masas e intercultural, con miras a mejorar las condiciones de salud de la población y los sistemas de salud.

- Tiene pertinencia social. La comunicación para la salud presta credibilidad y pertinencia a una disciplina que muchas veces se cuestiona en cuanto a utilidad pues, a pesar de que su foco de interés es la comunicación social, termina por crear un oscuro lenguaje propio, que dista mucho de hacerlo asequible a las personas ajenas a la especialidad. En las palabras de Gary Kreps, “Uno a veces se pregunta si la ciencia de las comunicaciones no terminará deteniéndose en seco, convertidos los comunicadores sociales en un grupo de ermitaños aislados, cada uno murmurándose a sí mismo palabras en un lenguaje que solo él puede entender" (8).

- Se ejerce generalmente en ámbitos interdisciplinarios. La naturaleza del quehacer en el área de la comunicación para la salud es por lo general interdisciplinaria $y$, por lo tanto, necesariamente opuesta al oscurantismo y la sobreespecialización (9). En los proyectos de comunicación para la salud confluyen conocimientos provenientes de la psicología social, sociología, medicina, epidemiología, antropología y salud pública, además de la comunicación social. Es común trabajar en equipos interdisciplinarios en los que hay que compartir experiencias y conocimientos prácticos de diferentes ámbitos para poder resolver problemas complejos. En estas circunstancias, es preciso usar lenguaje claro y poder poner en operación conceptos y teorías de comunicación social que sean útiles para el equipo interdisciplinario. 
El trabajo interdisciplinario suele ser beneficioso para todos los participantes. Los profesores investigadores de escuelas de comunicación frecuentemente trabajan en equipo con miembros de facultades de medicina para elaborar propuestas que han de presentarse a organismos que pueden financiarlas como los Institutos Nacionales de Salud (NIH) y fundaciones como las de Robert Wood Johnson, Kaiser Family Foundation, Kellogg y Wellness Foundation. Cabe notar que uno de los criterios más importantes para evaluar las propuestas es que los equipos que prometen desarrollar las campañas de promoción de la salud o de educación sobre tratamientos para enfermedades crónicas incluyan recursos humanos expertos en comunicación para la salud. Por excelente que parezca el proyecto y por muy ilustres que sean los investigadores médicos y epidemiológicos involucrados, si el proyecto no cuenta con un plan de comunicaciones bien pensado y con los recursos humanos que garanticen su instrumentación, el proyecto no consigue financiamiento (experiencia personal de la autora como miembro de comités de evaluación de proyectos, NIH).

Por otra parte, la colaboración con las escuelas de medicina hace posible que los investigadores en comunicaciones para la salud tengan acceso a recursos mucho mayores que los que generalmente pueden obtener. Las escuelas de comunicación suelen contar con escasos recursos para la investigación o la ejecución de campañas, especialmente cuando se trata de campañas de comunicación masiva en las comunidades, que son muy costosas. (La excepción a esta regla es la conocida Annenberg School of Communication, que cuenta con un benefactor privado, el señor Walter Annenberg.)

\section{Desarrollo de la comunicación para la salud en las universidades estadounidenses}

La comunicación para la salud penetró en el ámbito académico estadounidense por medio de profesores investigadores a quienes les interesaba ese campo en particular. Su trabajo en esta área de especialización ha sido básicamente autodidacta.

Actualmente en varios departamentos de comunicaciones se ofrecen programas de Magister "con énfasis en" comunicación para la salud, por ejemplo en la universidad Northwestern y las de South Florida, Maryland, Kentucky, Texas, Michigan State, Minnesota, Reno y California en Davis. Complementariamente, hay escuelas de salud pública que destacan la importancia de las comunicaciones en sus programas de Magister en Salud Pública. Entre estas figuran la Johns Hopkins School of Hygiene and Public Health, la Tulane School of Public Health y la Universidad de Maryland (9).
El programa de la Universidad de California en Davis. Hace 10 años, el Departamento de Comunicaciones de esta universidad no contaba con un programa de especialización en comunicaciones para la salud y tenía un enfoque muy pronunciado en la enseñanza de la retórica. Actualmente cuatro profesores investigadores trabajan en el área de la comunicación para la salud. El profesor Charles Berger, destacado teórico de la ciencia de la comunicación social, está estudiando la influencia de los medios masivos en la percepción del riesgo de enfermedades, concepto clave para el desarrollo de campañas de promoción de la salud. El profesor Mike Motley investiga las tácticas de comunicación interpersonal y de construcción de mensajes en situaciones problemáticas, por ejemplo, las de acoso sexual, violación entre personas conocidas y revelación de enfermedades de transmisión sexual. El profesor Robert Bell estudia los factores sociales que influyen en comportamientos conducentes a la prevención del sida y ha realizado estudios que analizan el contenido de la propaganda de productos farmacéuticos que aparece en revistas. Por último, la autora durante más de 20 años ha enfocado su investigación en el estudio de las audiencias y en la planificación, el diseño, la ejecución y la evaluación de campañas de comunicación dirigidas a poblaciones multiculturales. Esas campañas han abarcado la prevención del tabaquismo, la prevención de enfermedades del corazón, la planificación familiar, el cuidado prenatal y la vacunación.

Los cursos dictados por la facultad de comunicaciones de la Universidad de California en Davis atraen a alumnos de una gran variedad de campos de estudio, tales como educación, medicina, ciencias conductuales aplicadas, administración de empresas, sociología, agronomía, ciencias políticas y psicología. Tanto en Davis como en los departamentos de comunicaciones de otros centros de enseñanza se ofrecen cursos con títulos tales como comunicación y salud; teorías de persuasión y diseño de mensajes; comunicación grupal y negociación; teorías de comunicación masiva y promoción de la salud; diseño, ejecución y evaluación de campañas de comunicación; análisis y evaluación de audiencias; comunicación masiva y cambio social; y aspectos culturales de la promoción de la salud. Además de cursos como los mencionados, los programas de estudio requieren cursar estudios sobre metodologías de investigación en ciencias sociales, especialmente de investigación evaluativa.

El programa Emerson-Tufts de Magister en Comunicación para la Salud. Como ya se ha hecho notar, este programa conjunto del Departamento de Comunicaciones de la Universidad Emerson y la 
Escuela de Medicina de Tufts es el único que ofrece la posibilidad de obtener un título de posgrado en comunicación para la salud. El programa de estudios incluye series de asignaturas obligatorias y optativas. Las obligatorias cubren temas como estrategias éticas y efectivas de medios; comunicación profesional; métodos de investigación; teorías de comunicación, y persuasión y negociación. Además de las clases formales, el programa comprende contextos comunicacionales de manejo de crisis, presentaciones públicas que combinan diversos medios; conferencias de prensa y comunicación de grupos (1). Las asignaturas optativas permiten a los alumnos coordinar áreas de especialización en medicina, nutrición, salud pública, políticas sociales, mercadeo social, relaciones públicas, comunicación intercultural, promoción en salud, salud ambiental y ocupacional, salud maternoinfantil y salud internacional. El programa también exige prácticas en el terreno, ya sea en los medios de información y comunicación, instituciones gubernamentales o centros de salud.

Los alumnos de este programa no provienen exclusivamente de las profesiones liberales y del área de comunicaciones. Muchos pertenecen también a los campos de la medicina, la salud pública, la odontología, las ciencias veterinarias o la administración de empresas, y optan por un título que combina las dos áreas. El grado de magister, por lo tanto, puede otorgarse tanto a los alumnos que siguen el currículo de forma exclusiva como a los que prefieren un título combinado. Todos desarrollan las capacidades para planear, ejecutar y evaluar programas; servir de enlace entre los medios de comunicación, los expertos en salud, la comunidad y los funcionarios de instituciones de salud o de organismos gubernamentales, y diseminar información efectivamente (10).

Las posibles oportunidades de empleo para los graduados del programa de Emerson-Tufts incluyen los ámbitos del mercadeo social, las relaciones públicas, los servicios humanos, la salud comunitaria, la planificación y el desarrollo, el gobierno, la definición de políticas de nivel local, regional y nacional, y las organizaciones privadas. Hay además oportunidades de trabajo en los medios de comunicación, los sistemas privados de salud y los hospitales.

\section{CONCLUSIONES}

El interés en la comunicación para la salud como área de estudio en los Estados Unidos data de solo dos decenios. El desarrollo de este campo no ha sido sistemático, sino que ha respondido más bien de forma reactiva a la necesidad imperativa de incluir el aspecto de comunicaciones en la promoción de la salud. Pocas escuelas de comunicaciones tienen un plan de estudios dedicado al área de la comunicación para la salud y el aporte de los académicos ha sido principalmente de carácter individual dentro de las escuelas de comunicación social y de salud pública.

La comunicación para la salud es un área muy fecunda de trabajo y de investigación aplicada e interdisciplinaria. Representa un modelo de cómo la ciencia de las comunicaciones adquiere relevancia social aportando a otras áreas del quehacer humano —en este caso a la de la salud- teorías, conceptos y técnicas para mejorar el bienestar de la población. La promoción de la salud comprende una gran riqueza de conocimientos teóricos, investigación aplicada, modelos de acción y ejemplos de la aplicación práctica de los conocimientos sobre comunicaciones. Se espera que esta área de especialización tan importante comience su desarrollo en América Latina y el Caribe en un futuro muy cercano. Ello dependerá en gran medida de su introducción sistemática en escuelas de comunicación comprometidas con la investigación académica aplicada y la formación de generaciones futuras de especialistas en comunicación y salud, que puedan responder de forma adecuada al desafío de mejorar la calidad de vida en los países de las Américas.

Agradecimiento. La autora agradece a la OPS el respaldo brindado durante el presente trabajo.

\section{SYNOPSIS}

\section{Health communication as a field of study in universities in the United States of America}

This article analyzes the state of development of communications related to health in universities of the United States of America. This specialty is essential for people's wellbeing, and it involves interpersonal, organizational, and mass communications. In the United States, communications for health promotion is an area of applied communications with social relevance and generally performed in interdisciplinary settings. A number of universities in the United States offer communications master's degree programs with an emphasis on health. However, so far, the only program with a formal graduate degree in health and communications is one jointly offered by the Emerson University Department of Communications and the Tufts School of Medicine. Developing and including this specialization in the schools of communications in Latin America is crucial to improving the quality of life of the peoples of the continent. 


\section{REFERENCIAS}

1. Ratzan D, Stearns S, Payne G, Amato P, Liebergott J, Madoff M. Education for the health professional. En: Ratzan S, ed. Health communication: challenges for the $21^{\text {st }}$ century. Am Behav Sci 1994; 38(2):361-380.

2. Rogers E. The field of health communication today: an up-to-date report. J Health Commun 1996;1(1):15-23.

3. Rogers E. The field of health communication today. En: Ratzan S, ed. Health communication: challenges for the $21^{\text {st }}$ century. Am Behav Sci 1994;38(2):208-214.
4. Roper WL. Health communication takes on new dimensions at CDC. Public Health Rep 1993;108(2):179-183.

5. Ratzan S, Payne S, Bishop C. The status and scope of health communication. J Health Commun 1996;1(1):25-42.

6. Health reports. Time Magazine. September 1997:22.

7. McKinsey K. Hungarians' lifestyle is killing them. San Francisco Chronicle. October 1997.

8. Kreps G. Setting the agenda for health communication research and devel- opment: scholarship that can make a difference. Health Commun 1989;1(1): 11-15.

9. Kreps G, Thornton B. Health communication: theory and practice. 2a ed. Prospect Heights, IL: Waveland; 1992.

10. Maibach E, Parrott R, Long D, Salmon C. Competencies for the health communication specialists of the $21^{\text {st }}$ century. En: Ratzan S, ed. Health communication: challenges for the 21st century. Am Behav Sci 1994; 38(2):351-360. 